\title{
A Novel Elementary Particle Theory Based on External Energy Absorbed and Re-Emitted by Atoms
}

\author{
Hans W. Giertz \\ GiertzTech AB, Gnesta, Sweden \\ Email: hans@miklagaard.com \\ Received 27 January 2015; accepted 13 February 2015; published 25 February 2015 \\ Copyright (C) 2015 by author and Scientific Research Publishing Inc. \\ This work is licensed under the Creative Commons Attribution International License (CC BY). \\ http://creativecommons.org/licenses/by/4.0/ \\ (c) (i) Open Access
}

\begin{abstract}
In this study, all energy in the universe, here called energy quanta, originates from a singularity at the centre of the universe. The electron and the atom are completely passive; they absorb energy quanta by means of forced damped oscillators and the absorbed energy quanta are then re-emitted. Absorbed and stored energy quanta result in nuclear energy. Re-emitted energy quanta are fields. Re-emitted energy quanta operating on oscillators, in adjacent particles and atoms, result in forces: strong force, Coulomb force and gravitational force. Using this model may enable unification of elementary particle physics, general relativity, electromagnetic theory and quantum physics into one comprehensive theory.
\end{abstract}

\section{Keywords}

Electron, Atom, Gravity, Photon, Strong Force, Standard Model, Theory of Everything

\section{Introduction}

Today's elementary particle physics, quantum physics, electromagnetic theory and general relativity build on the assumption that electron and atomic forces, fields and energy are created or generated by the electron and atom itself without external influence. In the present study today's model is replaced by a fundamentally different model. In the new model all energy in the universe, here called energy quanta, originates from a singularity at the centre of the universe. The electron and the atom are completely passive; they absorb energy quanta by means of forced damped oscillators and the absorbed energy quanta are then re-emitted. Parts of this novel theory have been documented in eight different studies [1]-[8].

The Higgs Field is an energy field that exists everywhere in the universe and is accompanied by a fundamen- 
tal particle called the Higgs Boson [9]-[11]. It is proposed here that the Higgs Boson corresponds to one of the above energy quanta and that the Higgs Field corresponds to the matching energy quanta flow density.

Energy radiated from the singularity has been thoroughly measured and documented, including description of measurement technique [1] [2] and instrument technique [3]. It is a relatively simple task to repeat these measurements and to verify the existence of the singularity: its type of energy (energy quanta), its frequency spectrum and the direction towards the singularity. The direction to the singularity is towards north and along the earth's rotational axis [1] [2].

Atoms absorb energy quanta at frequencies specific to the atom. Thus, the atom contains (forced damped) oscillators with natural frequencies equal to the frequencies of energy quanta generated by the singularity. Each oscillator can be described as an oscillator quantum. The absorbed energy quanta result in nuclear forces and stored nuclear energy [1].

Electrons absorb energy quanta at a frequency specific to the electron. Thus, the electron contains an (forced damped) oscillator quantum with natural frequency equal to the frequency of energy quanta generated by the singularity. The absorbed energy quanta are called electron charge in today's physics. The absorbed energy quanta are then re-emitted uniformly into space by the oscillator quantum. Re-emitted energy quanta accounts for what is called Coulomb force, current, electric field, displacement and magnetic field in today's physics [12].

Atoms and electrons also absorb energy quanta at frequencies specific to gravity [2]. The absorbed energy quanta are re-emitted uniformly into space. The flow of energy quanta between oscillators in particles or masses results in mutual force of attraction, i.e. gravity [13]. It explains gravitational mass [5] [14].

Particle, e.g. electron excess energy $h v$ is superpositioned on energy quanta absorbed and re-emitted by oscillator quanta in the electron and the atomic nucleus [4]. Hence, the photon [15] [16] comprises superpositioned energy $h v$ on energy quanta, originating from the singularity. The conversion is made in the oscillator quantum [4]. It also explains inertial mass [5] [17] [18].

Parts of the present model have been bench marked on phenomena observed in astrophysics; it displays that it can replace general relativity [19]. The model provides simple solutions to metric expansion of space [6], gravitational lensing [7], gravitational redshift [8] and the black hole phenomena [8].

The present model is mapped on quantum physics and demonstrates solutions to intrinsic angular momentum, intrinsic magnetic dipole momentum and electron wave-particle duality [20] [21]. A solution to the Pauli Exclusion Principle is presented using injection locking [22] [23].

The purpose with the present paper is to provide a common theory for elementary particle physics, electromagnetic theory, quantum physics and astrophysics, i.e. a step towards the Theory of Everything (TOE). It complements and replaces parts of the Standard Model [24], electromagnetic theory [12], quantum physics [20] [21], general relativity [19] and string theory [25].

\section{Theoretical Model}

\subsection{General}

Today physics is described by different models: the Standard Model [24], electromagnetic theory [12], quantum physics [20] [21], general relativity [19] and string theory [25]. These theories have a common denominator. It is assumed that the characteristics of the electron and atomic nucleus, such as charge, fields, forces and energy, are intrinsic properties. Hence, today's physics claims that they are generated without external influence [12] [20]-[24]. The exception is the Higgs Field and the Higgs Boson [9]-[11].

This paper proposes that it is quite the opposite. The atom in itself contains no forces or energy, what so ever. The atom is completely passive. All energy in the universe originates from a singularity, positioned at the centre of the universe. This energy is absorbed by the electron and the atomic nucleus and the absorbed and re-emitted energy result in phenomena observed in physics: stored energy, forces, current and fields.

This section is structured in the following way. It begins with a model describing the electron's processes which create charge, Coulomb force, current, electric field and magnetic field. Only two quanta are involved: the energy quantum and the oscillator quantum. Both have a frequency specific to the electron.

The same model is then utilized to explain the nucleus strong force. The strong force is created by a process similar to the Coulomb force, albeit by energy quanta and oscillator quanta with frequencies specific to the nucleus and the strong force.

The same model is once again utilized to explain gravity. Gravity is created by a process similar to the Cou- 
lomb force, albeit by energy quanta and oscillator quanta with frequencies specific to gravity.

The same model is finally used to explain photons and inertial mass. Photons and inertial mass are created by a process where enforced energy $h v$ is superpositioned on energy quanta and where the mechanism enabling this conversion resides in oscillator quanta.

\subsection{Singularity}

According to the theoretical model the universe contains a singularity. This singularity has been described previously [1] [2] [4]-[8]. This singularity generates energy quanta at six different frequencies. The energy quantum has no mass and propagates with the speed of light. An energy quantum is described by its vector $\delta \boldsymbol{S}_{v}$ in the present study, where $v$ denotes its frequency $v$. The energy quantum $\delta \boldsymbol{S}_{v}$ remains unaltered as it travels through space. The singularity emits energy quanta $\delta \boldsymbol{S}_{v}$ uniformly into space creating energy flow density $\boldsymbol{S}_{v}(0)$ at the singularity and energy flow density $\boldsymbol{S}_{v}(R)$ at the distance $R$. Energy quanta $\delta \boldsymbol{S}_{v}$ are radiated uniformly into space, which results in that $\boldsymbol{S}_{v}(R)$ decreases with the area of the sphere

$$
\boldsymbol{S}_{v}(R)=\boldsymbol{S}_{v}(R=0) \frac{1}{4 \pi R^{2}} .
$$

\subsection{Electron Charge}

This section describes the process which results in electron charge and Coulomb force. The process is general and is, in Sections 2.7, 2.8 and 2.9, utilized to explain positron, nucleus and gravitational forces. The electron contains an (forced damped) oscillator with natural frequency $v_{e}$ which absorbs energy quanta $\delta \boldsymbol{S}_{v_{e}}$ and energy flow density $S_{v_{e}}(R)$ with frequency $v_{e}$, originating from the singularity. $e$ denotes electron. The oscillator can be described as an oscillator quantum. The absorbed energy quanta $\delta \boldsymbol{S}_{v_{e}}$ are re-emitted and distributed uniformly into space as energy flow density $\boldsymbol{S}_{v_{e}}(r)$ at the distance $r$ from the electron. Hence, the energy flow density emitted by the electron is

$$
\boldsymbol{S}_{v_{e}}(r)=\alpha \boldsymbol{S}_{v_{e}}(R=0) \frac{1}{4 \pi R^{2}} \cdot \frac{1}{4 \pi r^{2}},
$$

where $\alpha$ is a constant which mirrors the oscillator quantum transfer function. However, at the earth the distance $R$ to the singularity is constant. Equation (2) can, thus, be simplified into

$$
\boldsymbol{S}_{v_{e}}(r)=\boldsymbol{S}_{v_{e}}(r=0) \frac{1}{4 \pi r^{2}} \text {. }
$$

From now on $S_{v_{e}}(r=0)$ is denoted $S_{v_{e}}(0)$. Equation (3) describes the energy flow density from one electron. Now one electron is positioned at the distance $r$ from a second electron. There is a mutual flow of energy quanta $\delta \boldsymbol{S}_{v_{e}}$ and energy flow density $\boldsymbol{S}_{v_{e}}(r)$ between the two electron's oscillator quantum, and where the energy flow density is described by Equation (3). Energy quanta $\delta \boldsymbol{S}_{v_{e}}$ propagating in opposite directions between the two electrons results in the repelling force $\delta \boldsymbol{F}_{v_{e}}$ on each electron's oscillator quantum

$$
\delta \boldsymbol{F}_{v_{e}}(r)=\delta \boldsymbol{S}_{v_{e}} \cdot \delta \boldsymbol{S}_{v_{e}} .
$$

Energy flow density $\boldsymbol{S}_{v_{e}}(r)$ propagating in opposite directions between an electron positioned at the distance $r$ and an electron positioned at $r=0$ results in a repelling force $\boldsymbol{F}_{v_{e}}(r)$ on each electron's oscillator quantum

$$
\boldsymbol{F}_{v_{e}}(r)=\boldsymbol{S}_{v_{e}}(r) \cdot \boldsymbol{S}_{v_{e}}(0),
$$

and which according to Equation (3) can be re-written into

$$
\boldsymbol{F}_{v_{e}}(r)=S_{v_{e}}(0) \cdot S_{v_{e}}(0) \frac{\boldsymbol{r}}{4 \pi r^{3}} .
$$

Equation (6) can be compared with classical electromagnetic theory and Coulomb's law for two electrons with elementary charge $e$ [12]

$$
\boldsymbol{F}_{\text {Coulomb }}(r)=\frac{e \cdot e}{4 \pi \varepsilon_{0}} \frac{\boldsymbol{r}}{r^{3}}
$$


and where we see that $S_{v_{e}}(0)$ corresponds to the classical definition of the electron elementary charge $e$, $1.6019 \times 10^{-19}$ coulombs. $\varepsilon_{0}$ denotes that Equation (7) has been calculated using the electric field $\boldsymbol{E}$ instead of displacement $\boldsymbol{D}, \boldsymbol{D}=\varepsilon_{0} \boldsymbol{E}$ (see Section 2.5). Equation (6) can be modified to include clusters of $k$ electrons with energy flow density $\sum_{1}^{k} S_{v_{e}}(r)$ or $\iint S_{v_{e}}(r)$ and which is proportional to the number of oscillator quanta involved. $\sum_{1}^{k} S_{v_{e}}(0)$ corresponds to the charge $Q_{1}$ or $Q_{2}$. This corresponds to Coulomb's law for two charges at distance $r$ [12]

$$
\boldsymbol{F}_{\text {Coulomb }}(r)=\frac{Q_{1} Q_{2}}{4 \pi \varepsilon_{0}} \frac{\boldsymbol{r}}{r^{3}}
$$

\subsection{Electron Current}

Current $\boldsymbol{i}_{v_{e}}$ is the transport with velocity $\boldsymbol{v}$ of the re-emitted energy flow density $S_{v_{e}}(0)$ from $n$ electrons:

$$
\boldsymbol{i}_{v_{e}}=-n \cdot S_{v_{e}}(0) \cdot \boldsymbol{v},
$$

where the "-" sign denotes that the definition of current flow is opposite to the electron transport direction. Equation (9) can be compared with the classical definition of current [12]

$$
\boldsymbol{i}=-n \cdot e \cdot v,
$$

where $S_{v_{e}}(0)$ corresponds to the elementary charge $e$ as described in Section 2.3.

\subsection{Electron Magnetic Field}

In the present study magnetism is described in the following way. An electron, with velocity $\boldsymbol{v}$, passes a fixed point $(x, y, z)$ in a given reference system. The electron re-emits the energy flow density $\boldsymbol{S}_{v_{e}}(r)$. When the electron passes the fixed point $(x, y, z)$ the derivate of $\boldsymbol{S}_{v_{e}}(r)$ is observed, i.e. $\frac{\partial \boldsymbol{S}_{v_{e}}(r)}{\partial t}$. Perpendicular to the direction of $\boldsymbol{v}$, the derivate $\frac{\partial \boldsymbol{S}_{v_{e}}(r)}{\partial t}$ forms equipotential surfaces, called $\boldsymbol{H}_{v_{e}}(r)$ at the distances $r$. Each equipotential surface is continuous; hence it is described by its curl

$$
\operatorname{curl} \boldsymbol{H}_{v_{e}}(r)=\frac{\partial \boldsymbol{S}_{v_{e}}(r)}{\partial t} .
$$

Equation (11) describes $\boldsymbol{H}_{v_{e}}(r)$ resulting from one electron. $\boldsymbol{H}_{v_{e}}^{n}(r)$ from $n$ electrons propagating with the velocity $\boldsymbol{v}$ is then described by combining Equation (9) and Equation (11)

$$
\operatorname{curl} \boldsymbol{H}_{v_{e}}^{n}(r)=\boldsymbol{i}_{v_{e}} \text {. }
$$

Now we picture current $\boldsymbol{i}_{v_{e}}$ propagating in a wire. The wire is cut and at this place a capacitor is positioned. Electrons accumulate on each capacitor plate and each electron on one plate results in energy flow density $\boldsymbol{S}_{v_{e}}(r)$ according to Equation (3). This energy flow density and its derivate also form equipotential surfaces. $n$ electrons result in energy flow density $\boldsymbol{S}_{v_{e}}^{n}(r)$ which is the vector sum of the energy flow density from $n$ contributing electrons at the distance $r$ from the capacitor plate. Hence, $\boldsymbol{H}_{v_{e}}^{n}(r)$ at the distance $r$ from the capacitor plate is

$$
\operatorname{curl} \boldsymbol{H}_{v_{e}}^{n}(r)=\frac{\partial \boldsymbol{S}_{v_{e}}^{n}(r)}{\partial t} .
$$

Consequently, $\boldsymbol{H}_{v_{e}}^{n}(r)$ resulting from current $\boldsymbol{i}_{v_{e}}$ propagating in the wire and energy flow density $\boldsymbol{S}_{v_{e}}^{n}(r)$ propagating in vacuum (in the capacitor) is

$$
\operatorname{curl} \boldsymbol{H}_{v_{e}}^{n}(r)=\boldsymbol{i}_{v_{e}}+\frac{\partial \boldsymbol{S}_{v_{e}}^{n}(r)}{\partial t} .
$$

$\boldsymbol{H}_{v_{e}}^{n}(r)$ can be defined as the magnetic field, similar to the magnetic field $\boldsymbol{H}$ in classical electromagnetic theory. Equation (14) corresponds to Maxwell's equation [12] 


$$
\operatorname{curl} \boldsymbol{H}=\boldsymbol{i}+\frac{\partial \boldsymbol{D}}{\partial t} .
$$

$\boldsymbol{S}_{v_{e}}^{n}(r)$ corresponds to the displacement $\boldsymbol{D}$ in classical electromagnetism and $\boldsymbol{S}_{v_{e}}(r)$ corresponds to the displacement $\boldsymbol{D}$ from one electron (i.e. from one oscillator quantum). According to classical electromagnetism $\boldsymbol{D}=\varepsilon_{0} \boldsymbol{E}$ (in vacuum). Thus, the electric field $\boldsymbol{E}$ corresponds to $\frac{\boldsymbol{S}_{v_{e}}^{n}(r)}{\varepsilon_{0}}$. However, $\boldsymbol{D}$ and $\boldsymbol{E}$ are defined as static fields in classical electromagnetism. In the present theory they consist of energy quanta with frequency $v_{e}$.

\subsection{Revised Electromagnetic Theory}

It is proposed that the electromagnetic theory can be revised and simplified. The revised version includes energy quantum $\delta \boldsymbol{S}_{v_{e}}$, oscillator quantum with frequency $v_{e}$, energy flow density from one electron $\boldsymbol{S}_{v_{e}}(r)$, energy flow density from $n$ electrons $\boldsymbol{S}_{v_{e}}^{n}(r)$ (displacement $\boldsymbol{D}$ ), force $\boldsymbol{F}_{v_{e}}(r)$ according to Equation (5), current $\boldsymbol{i}_{v_{e}}$ according to Equation (9), magnetic field $\boldsymbol{H}_{v_{e}}^{n}(r)$ according to Equation (14) and voltage $V(\boldsymbol{E}=-\operatorname{grad} V)$. The electric field $\boldsymbol{E}$ can be replaced with ${ }^{\boldsymbol{D}}=\varepsilon \varepsilon_{0} \boldsymbol{E}$. The magnetic field $\boldsymbol{B}_{v_{e}}^{n}(r)$ can be replaced with $\boldsymbol{B}_{v_{e}}^{n}(r)=\mu \mu_{0} \boldsymbol{H}_{v_{e}}^{n}(r)$. Maxwell's equations are still valid, provided that they are modified using energy flow density and its time derivate according to the above description.

Sections 2.7, 2.8 and 2.9 explain how the revised electromagnetic theory can be utilized to describe the positron, the nucleus, gravity and astrophysics. The revised electromagnetic theory is general and forms the fundament for unification of many scientific disciplines.

\subsection{Positron}

This section explains that the positron is identical to the electron; however, the positron energy quantum and oscillator quantum have a frequency specific to the positron. The positron contains an (forced damped) oscillator quantum with natural frequency $v_{p}$ which absorbs energy quanta $\delta \boldsymbol{S}_{v_{p}}$ and energy flow density $\boldsymbol{S}_{v_{p}}(R)$, and where $p$ denotes positron. Thus, the positron frequency is different to the electron frequency. The absorbed energy consists of energy quanta $\delta \boldsymbol{S}_{v_{p}}$ which are re-emitted and distributed uniformly into space as energy flow density $S_{v_{p}}(r)$ at the distance $r$ from the positron. The rules and laws of the positron are identical to those of the electron, except that its frequency is $v_{p}$. There is a repelling force caused by energy flow density $\boldsymbol{S}_{v_{p}}(r)$ between two positrons

$$
\boldsymbol{F}_{p_{p}}(r)=\boldsymbol{S}_{v_{p}}(0) \cdot \boldsymbol{S}_{v_{p}}(0) \frac{\boldsymbol{r}}{4 \pi r^{3}} .
$$

Hence, energy flow density between oscillators having exactly the same frequency results in repelling force. However, energy flow density between oscillators having slightly different frequencies results in attracting force.

The electron and the positron have slightly different frequencies. Thus, there is a force of attraction $\boldsymbol{F}_{e_{p}}(r)$ between energy flow density $S_{v_{e}}(r)$ from an electron operating on the positron oscillator quantum with frequency $v_{p}$. Likewise, there is a force of attraction $\boldsymbol{F}_{e_{p}}(r)$ between energy flow density $\boldsymbol{S}_{v_{p}}(r)$ from a positron operating on the electron oscillator quantum with frequency $v_{e}$.

$$
\boldsymbol{F}_{e_{p}}(r)=-\boldsymbol{S}_{v_{e}}(0) \cdot \boldsymbol{S}_{v_{p}}(0) \frac{\boldsymbol{r}}{4 \pi r^{3}},
$$

where the “-” sign denotes force of attraction. Consequently, the positron applies to revised electromagnetic theory and revised Maxwell's equations provided parameters include the positron frequency $v_{p}$.

\subsection{Strong Force and Nuclear Energy}

This section explains that the atomic nucleus contains mechanisms similar to the positron and electron; however, the nucleus energy quanta and oscillator quanta have frequencies specific to the nucleus. Neutrons and protons contain large numbers of oscillator quanta with two slightly different frequencies $v_{n 1}$ and $v_{n 2}$, where $n$ denotes nucleus and its strong force. This creates energy flow density between oscillator quanta as described in Sections 2.3 and 2.7. The energy flow density results in force of attraction $\boldsymbol{F}_{v_{n 12}}(r)$ between oscillator quanta with 
slightly different frequencies, $v_{n 1}$ and $v_{n 2}$, within fermions, within neutrons, within protons and between neutrons and protons.

$$
\boldsymbol{F}_{v_{n 12}}(r)=-\boldsymbol{S}_{v_{n 1}}(r) \cdot \boldsymbol{S}_{v_{n 2}}(0) \text {. }
$$

It also creates repelling forces $\boldsymbol{F}_{v_{n 11}}(r)$ and $\boldsymbol{F}_{v_{n 22}}(r)$ between oscillator quanta with equal frequency $v_{n 1}$ or $v_{n 2}$

$$
\boldsymbol{F}_{v_{n 11}}(r)=\boldsymbol{S}_{v_{n 1}}(r) \cdot \boldsymbol{S}_{v_{n 1}}(0) \text {. }
$$

It is proposed that $\boldsymbol{F}_{v_{n 12}}(r)$ is larger than $\boldsymbol{F}_{v_{n 11}}(r)$ and $\boldsymbol{F}_{v_{n 22}}(r)$, creating a net force of attraction. This implies that the strong force between oscillators in the nucleus is similar to the force between electrons and positrons, except the difference in frequencies. It is proposed that oscillators in the nucleus are not static; they oscillate and orbit each other similar to electrons orbiting the nucleus. Fermions, neutrons and protons contain very large numbers of oscillator quanta; consequently, the combined forces result in very strong forces. The oscillators are densely packed, resulting in that re-emitted energy density $\boldsymbol{S}_{v_{n 1}}(r)$ and $\boldsymbol{S}_{v_{n 2}}(r)$ are primarily absorbed by oscillators within the nucleus and minor energy flow density outside the nucleus. This also implies that the energy flow density within the nucleus is very large. This results in stored energy, i.e. nuclear energy and binding energy within fermions, within neutrons, within protons and between neutrons and protons.

It is proposed that elementary particles like fermions, neutrons and protons are different configurations of oscillators with frequency $v_{n 1}, v_{n 2}, v_{e}, v_{p}, v_{g 1}$ and $v_{g 2}$, where $v_{g 1}$ and $v_{g 2}$ are described in Section 2.9. Bosons are energy quanta with frequency $v_{n 1}, v_{n 2}, v_{e}, v_{p}, v_{g 1}$ and $v_{g 2}$.

The implication of this is that the atomic nucleus can and should be described by the same laws as the revised electromagnetic theory, except that the frequencies are different $\left(v_{n 1}\right.$ and $\left.v_{n 2}\right)$. Consequently, the nucleus is described by the nucleus energy quantum vectors $\delta \boldsymbol{S}_{v_{n}}$, nucleus energy flow density from one oscillator $\boldsymbol{S}_{v_{n}}(r)$, nucleus energy flow density from $n$ oscillators $\boldsymbol{S}_{v n}^{n}(r)$ (nucleus displacement $\boldsymbol{D}_{v_{n}}$ ), nucleus force $\boldsymbol{F}_{v_{n}}(r)$ according to Equation (5), nucleus current $\boldsymbol{i}_{v_{n}}$ according to Equation (9), and nucleus magnetic field $\boldsymbol{H}_{v_{n}}^{n^{n}}(r)$ according to Equation (14). The nucleus is not static; oscillators in fermions, neutrons and protons orbit and oscillate. This creates internal displacement $\boldsymbol{D}_{v_{n}}$ and magnetic field $\boldsymbol{H}_{v_{n}}^{n}(r)$ which may result in what is called the nuclear weak force. The atomic nucleus applies to the revised electromagnetic theory, provided parameters include the nucleus frequencies $v_{n 1}, v_{n 2}, v_{e}, v_{p}, v_{g 1}$ and $v_{g 2}$.

\subsection{Gravity}

This section explains that gravity is created by mechanisms similar to the electron, positron and the atomic nucleus; however, gravity encompasses energy quanta and oscillator quanta with frequencies specific to gravity. Gravity has been described in detail in [2]. Neutrons, protons and electrons contain oscillator quanta with natural frequencies $v_{g 1}$ and $v_{g 2}$, where $g$ denotes gravity. The frequencies $v_{g 1}$ and $v_{g 2}$ are slightly different. This creates energy flow density between oscillator quanta as described in Sections 2.3 and 2.7. The energy flow density creates force of attraction $\delta \boldsymbol{F}_{v_{g 12}}(r)$ between oscillator quanta in atoms and electrons having slightly different frequencies $v_{g 1}$ and $v_{g 2}$

$$
\delta \boldsymbol{F}_{v_{g 12}}(r)=-\boldsymbol{S}_{v_{g 1}}(r) \cdot \boldsymbol{S}_{v_{g 2}}(0) \text {. }
$$

The force of attraction $\boldsymbol{F}_{v_{g 12}}(r)$ between two bodies containing $n$ respectively $p$ oscillator quanta is

$$
\boldsymbol{F}_{v_{g 12}}(r)=-n \boldsymbol{S}_{v_{g 1}}(r) \cdot p \boldsymbol{S}_{v_{g 2}}(0) \text {. }
$$

It is assumed that that the distance $r$ between the bodies is much larger than the distance between oscillator quanta within each body. Equation (21) can be re-written into

$$
\boldsymbol{F}_{v_{g 12}}(r)=-\gamma n p \cdot \boldsymbol{S}_{v_{g 1}}(0) \cdot \boldsymbol{S}_{v_{g 2}}(0) \frac{\boldsymbol{r}}{4 \pi r^{3}}=-\eta n p \cdot \frac{\boldsymbol{r}}{4 \pi r^{3}},
$$

where $\gamma$ and $\eta$ are constants. It also creates repelling forces $\boldsymbol{F}_{v_{g 11}}(r)$ and $\boldsymbol{F}_{v_{g 22}}(r)$ between oscillator quanta with equal frequency $v_{g 1}$ or $v_{g 2}$

$$
\boldsymbol{F}_{v_{g 11}}(r)=\xi \gamma n p \cdot \boldsymbol{S}_{v_{g 1}}(0) \cdot \boldsymbol{S}_{v_{g 1}}(0) \frac{\boldsymbol{r}}{4 \pi r^{3}}=\xi \eta n p \cdot \frac{\boldsymbol{r}}{4 \pi r^{3}} .
$$


$\xi$ denotes that the repelling forces $\boldsymbol{F}_{v_{g 11}}(r)$ and $\boldsymbol{F}_{v_{g 22}}(r)$ are smaller than $\boldsymbol{F}_{v_{g 12}}(r)$. This creates a net force of attraction $\boldsymbol{F}_{g}(r)$. $\xi$ can be described as $1-\Delta$, where $\Delta$ is extremely small. This explains why the gravitational force $\boldsymbol{F}_{g}(r)$ is extremely small.

The number of oscillator quanta $n$ in body 1 respectively $p$ in body 2 is proportional to the gravitational mass $m_{1}$ of body 1 and the gravitational mass $m_{2}$ of body 2 . We now recognize the similarity with Newton's metric law of gravity, where $G$ is Newton's gravitational constant [13].

$$
F_{g}(r)=\Delta \eta n p \cdot \frac{1}{4 \pi r^{2}} \approx F_{\text {Newton }}=G m_{1} m_{2} \frac{1}{r^{2}} .
$$

We also recognize the similarity with Coulomb's law $F_{\text {Coulomb }}(r)=\frac{Q_{1} Q_{2}}{4 \pi \varepsilon_{0} r^{2}}$. The reason is that the gravitational force is similar to the force between clusters of electrons. The number of involved oscillator quanta in atoms and the frequencies $v_{g 1}$ and $v_{g 2}$ determines the gravitational constant $\Delta \eta$.

The implication of this is that the solar system, the Milky Way Galaxy and the universe in general should be described by the same laws as the revised electromagnetic theory, except that the frequencies are different $\left(v_{g 1}\right.$ and $v_{g 2}$ ). Hence, the relationship between planets, stars and galaxies is described by the gravitational energy quantum vector $\delta \boldsymbol{S}_{v_{g}}$, gravitational energy flow density from one oscillator quantum $\boldsymbol{S}_{v_{q}}(r)$, gravitational energy flow density from $n$ oscillator quanta $\boldsymbol{S}_{v_{g}}^{n}(r)$ (gravitational displacement $\boldsymbol{D}_{v_{q}}$ ), gravitational force $\boldsymbol{F}_{v_{g}}(r)$ according to Equation (5), gravitational current $\boldsymbol{i}_{v_{g}}$ according to Equation (13), and gravitational magnetic field $\boldsymbol{H}_{v_{g}}^{n}(r)$ according to Equation (14). Hence, all bodies (planets, stars) in the universe generate gravitational current, gravitational force, gravitational displacement and gravitational magnetic field. They apply to the revised electromagnetic theory, provided the parameters include the gravitational frequencies $v_{g 1}$ and $v_{g 2}$.

\subsection{Photon}

The nature of the photon has been described in detail [4]. The process creating photons is described here in condensed form. Particles, e.g. electron, neutron and proton contain one or many oscillator quanta with natural frequency $v_{g}$ creating gravity as described in Section 2.9. Each oscillator quantum absorbs energy quanta $\delta \boldsymbol{S}_{v g}$ and re-emits the energy quanta $\delta \boldsymbol{S}_{v_{g}}$. Accelerating an electron implies that force and energy is enforced on the electron. This energy can be described as many energy quanta $h v$. The energy quanta $h v$ cannot be stored in the electron and its oscillator quanta. The energy quanta $h v$ are, as a result, superpositioned on re-emitted energy quanta $\delta \boldsymbol{S}_{v_{g}}$. One energy quantum $h v$ is superpositioned (in the oscillator quantum) on one re-emitted energy quantum $\delta \boldsymbol{S}_{v_{g}}$ at a time. This new energy quantum $\delta \boldsymbol{S}_{v_{g+v}}$ has the frequency $v_{g}$ and $v$, where $v_{g}$ can be either $v_{g 1}$ or $v_{g 2}$. Likewise, decelerating an electron implies that force and energy is enforced on the electron. The energy quanta $h v$ are, therefore, superpositioned on re-emitted energy quanta in exactly the same way as when electrons are accelerated. Radio waves, light and gamma rays are energy quanta $h v$ superpositioned on gravity energy quanta $\delta \boldsymbol{S}_{v_{g}}$.

\subsection{Gravitational and Inertial Mass}

Gravitational and inertial mass have been described in detail [5]. The process behind gravitational and inertial mass is described here in condensed form.

Gravitational mass is the expression of the mechanism, i.e. atomic oscillator quanta which absorb and re-emit energy quanta $\delta \boldsymbol{S}_{v_{g}}$ and energy flow density $\boldsymbol{S}_{v_{g}}(r)$. This results in gravitational force according to Equations (22) and (23). Consequently, the gravitational mass $m_{1}$ in Equation (24) is proportional to the number $n$ of oscillator quanta with natural frequency $v_{g}$ (creating gravity) in the mass or the body.

Accelerating or decelerating a mass, a particle, an electron or an atom demands enforced force and energy. The mass, particle, electron or atom cannot store this enforced energy. The enforced energy can be described as energy quanta $h v$. The enforced energy quanta $h v$ are superpositioned on absorbed and re-emitted energy quanta $\delta \boldsymbol{S}_{v_{q}}$ as described in Section 2.10. Hence, the enforced energy quanta $h v$ are emitted as photons with energy $h v$. Thus, inertial mass is the expression of the mechanism, i.e. atomic oscillator quanta with natural frequency $v_{g}$, which convert enforced energy quanta $h v$ into emitted photons with energy $h v$. The inertial mass is proportional to the number $n$ of oscillator quanta (creating gravity) in the mass. Consequently, the inertial mass is propor- 
tional to the gravitational mass, although gravitational and inertial mass originate from totally different processes. Hence, the process creating inertial mass is identical to the process creating photons, as described in Section 2.10.

\subsection{Quantum Physics}

In this section it is assumed that the electron comprises three oscillator quanta, each one with frequencies $v_{e}, v_{g 1}$ and $v_{g 2}$ (see Section 3). This simple configuration of three oscillator quanta provides the electron with intrinsic characteristics described in Sections 2.3, 2.4, 2.5, 2.9, 2.10 and 2.11. The oscillator quanta $v_{e}, v_{g 1}$ and $v_{g 2}$ do not comprise a static configuration. They oscillate and orbit each other.

The electron has intrinsic angular momentum (up spin and down spin) and intrinsic magnetic dipole momentum, according to quantum physics [20] [21]. The oscillator quanta $v_{e}, v_{g 1}$ and $v_{g 2}$ orbit each other, i.e. they have spin. The energy quanta $\delta \boldsymbol{S}_{v_{e}}, \delta \boldsymbol{S}_{v_{q 1}}$ and $\delta \boldsymbol{S}_{v_{q 2}}$ are polarized [1] [2] [4]. This polarization results in that the oscillator quanta spin is oriented either 0 degree or 180 degrees relative to the absorbed energy quanta. This is called up spin and down spin. The spin implies that all three oscillator quanta accelerate in their orbits. It results in both magnetic fields $\boldsymbol{H}_{v_{e}}, \quad \boldsymbol{H}_{v_{g 1}}$ and $\boldsymbol{H}_{v_{q 2}}$, as described in Section 2.5 and magnetic dipole momentums.

The electron has a wave function and applies to the wave-particle duality [20] [21]. Sections 2.3 and 2.9 describe that the electron absorbs and re-emits energy quanta $\delta \boldsymbol{S}_{v_{e}}, \delta \boldsymbol{S}_{v_{g 1}}$ and $\delta \boldsymbol{S}_{v_{g 2}}$. The re-emitted energy flow density $\boldsymbol{S}_{v_{e}}$ is described in Equation (3). This defines the electrons characteristics. Consequently, the electron and its oscillator quanta is primarily a wave function comprising the frequencies $v_{e}, v_{g 1}$ and $v_{g 2}$. Thus, the electron can be described as a particle consisting of its oscillator quanta as well as a wave consisting of re-emitted energy quanta. The electron's wave function explains why electrons orbiting the nucleus exhibit discrete orbits. The electron's permitted orbits, with radius $r$, are related to the electron's wavelength $\lambda_{e}=n 2 \pi r$, where $\lambda_{e}=1 / v_{e}$ and $n=1,2,3, \cdots$.

The electron is exposed to forces between the electron and the singularity as well as forces between the electron and adjacent electrons and protons. These forces are caused by absorbed energy quanta with frequencies $v_{e}$, $v_{g 1}$ and $v_{g 2}$, as described in Equations (6), (17), (22) and (23). These forces are not static and, consequently, the electron's position will oscillate with the frequencies $v_{e}, v_{g 1}$ and $v_{g 2}$. This may shed light on the uncertainty principle [20] [21].

According to classical and quantum physics an orbiting (accelerated) electron radiates energy (photons) [20] [21]. Hence, it loses energy and should, consequently, rapidly approach and collide with the nucleus. However, electrons have fixed orbits. The electron absorbs energy quanta from the singularity and which compensates lost energy when the electron is accelerated in its orbit. Hence, there is energy balance which ensures fixed orbits.

The Pauli Exclusion Principle says that two fermions (e.g. two electrons) cannot occupy the same quantum state [20] [21]. This is illustrated with electrons orbiting the nucleus. In order to understand the Pauli Exclusion Principle injection locking is introduced [22] [23]. Injection locking is the frequency effects that can occur when an oscillator is disturbed (or frequency shifted) by injected energy. This implies that the electron oscillator quantum frequency $v_{e}$ is slightly altered by energy (photons) generated when the electron is accelerated in its orbit (see Section 2.10). Altering the frequency $v_{e}$ results in that the force of attraction $\boldsymbol{F}_{e_{p}}(r)$, Equation (17), between electron and nucleus is slightly reduced, whereby the electron is forced into an orbit with a different radius. This orbit is stable when there is equilibrium between acceleration, $v_{e}, \boldsymbol{F}_{e_{p}}(r)$ and when $\lambda_{e}=n 2 \pi r$ is fulfilled. Hence, the electron can have many stable and discrete orbits. In principle the radius can vary with time, resulting in diffuse; however, stable orbits. The repelling force $\boldsymbol{F}_{v_{e}}$, Equation (6), between orbiting electrons is minimized when they have different states, i.e. different orbits. Hence, each orbit can be occupied by only one electron with the same quantum state, i.e. one electron with up spin and one with down spin.

Consequently, phenomena observed in quantum physics can be explained with the present model.

\section{Elementary Particles}

In this section it is assumed that the universe is organized in a simple and logical way. It is proposed that the universe, outside the singularity, contains only two types of primary entities. The two primary entities are the energy quantum $\delta \boldsymbol{S}_{v}$ and the atomic/particle oscillator quantum with natural frequency $v$. They are the two universal quanta. They can have six different frequencies; $v_{e}, v_{p}, v_{n 1}, v_{n 2}, v_{g 1}$ and $v_{g 2}$. Each frequency provides unique characteristics to the electron, positron, nucleus, gravity and energy transport in space. 
The six frequencies are arranged in the frequency spectrum in the following order: $v_{n 1}, v_{n 2}, v_{g 1}, v_{g 2}, v_{p}, v_{e}$, where $v_{n 1}$ has the highest frequency. These frequencies are very close to each other which results in that there is a force of attraction between oscillator quanta $v_{n 1}, v_{n 2}, v_{g 1}$ and $v_{g 2}$ as described in Equation (17). It is proposed that these forces tend to unite oscillator quanta into stable triplets of oscillator quanta, where one triplet consists of $v_{n 1}, v_{g 1}$ and $v_{g 2}$ and the other triplet consists of $v_{n 2}, v_{g 1}$ and $v_{g 2}$. The frequencies $v_{g 1}, v_{g 2}, v_{p}, v_{e}$ are also close to each other. It is proposed that it results in one triplet $v_{p}, v_{g 1}$ and $v_{g 2}$ (positron) and one triplet $v_{e}$, $v_{g 1}$ and $v_{g 2}$ (electron). There could, of course, be other configurations, e.g. $v_{n 1}, v_{n 1}, v_{g 1}$ and $v_{g 2}$. However, the frequencies $v_{n 1}$ and $v_{e}$ are too separated to form a bond according Equation (17). Thus, only some configurations are feasible. In the following a very simple structure is proposed. The purpose is to show that the atom may have simple and repetitive structures. It is not intended as a solution; however, a guideline showing possible principles.

In its simplest configuration the universe may consists of only four basic particles: electron $\left(v_{e}, v_{g 1}, v_{g 2}\right)$, positron $\left(v_{p}, v_{g 1}, v_{g 2}\right)$ and two nuclear basic particles called $\alpha$-tron $\left(v_{n 1}, v_{g 1}, v_{g 2}\right)$ and $\beta$-tron $\left(v_{n 2}, v_{g 1} . v_{g 2}\right)$. The $\alpha$-tron and $\beta$-tron are the enablers of the strong force and nuclear energy. All four basic particles have almost identical structures. They contain one common part $\left(v_{g 1}, v_{g 2}\right)$ resulting in gravity, gravitational mass, inertial mass and production of photons. They also contain a second individual part consisting of one of the oscillator quanta $v_{n 1}$, $v_{n 2}, v_{p}$ or $v_{e}$. This part results in the "positive" positron $\left(v_{p}\right)$, the "negative" electron $\left(v_{e}\right)$, the "positive" $\alpha$-tron $\left(v_{n 1}\right)$ and the "negative" $\beta$-tron $\left(v_{n 2}\right)$. Thus, the four basic particles are identical except for one parameter; the frequencies $v_{n 1}, v_{n 2}, v_{p}$ and $v_{e}$. The frequency parameter determines the characteristics of the different parts of the atom. Configurations of $\alpha$-trons, $\beta$-trons, positron and electron constitute neutron, proton and other particles.

Perceived particle mass is always the result of oscillator quanta $v_{g 1}, v_{g 2}$ and their re-emitted energy quanta $\delta \boldsymbol{S}_{v_{g}}$. All forces are created by the oscillator quanta $v_{e}, v_{p}, v_{n 1}, v_{n 2}, v_{g 1}, v_{g 2}$ and their re-emitted energy quanta. All nuclear energy is created by the oscillator quanta $v_{e}, v_{p}, v_{n 1}, v_{n 2}, v_{g 1}, v_{g 2}$ and absorbed and re-emitted energy quanta. Fermions contain oscillator quanta. Bosons are energy quanta $\delta \boldsymbol{S}_{v}$ emitted by the singularity, absorbed by oscillator quanta in particles or re-emitted by oscillator quanta in particles.

The present model separates between the entity which enables fields, forces, stored energy and emitted energy (photons) on one hand and the entity which mediates fields, forces, stored energy and emitted energy on the other hand. The enablers of fields, forces, stored energy and emitted energy are the oscillator quanta $v_{e}, v_{p}, v_{n 1}$, $v_{n 2}, v_{g 1}$ and $v_{g 2}$. The mediators of fields, forces, stored energy and emitted energy are the energy quanta $\delta \boldsymbol{S}_{v}$ with frequencies $v_{e}, v_{p}, v_{n 1}, v_{n 2}, v_{g 1}$ and $v_{g 2}$.

\section{Discussion and Conclusions}

The present study proposes that all energy in the universe originates from a singularity at the centre of the universe. The universe, outside the singularity, contains only two types of primary entities. They are the energy quantum $\delta \boldsymbol{S}_{v}$ and the atomic/particle oscillator quantum with natural frequency $v$. They can have six different frequencies; $v_{e}, v_{p}, v_{n 1}, v_{n 2}, v_{g 1}$ and $v_{g 2}$. Configurations of oscillator quanta with different natural frequencies constitute particles such as fermions, electron, positron, neutron and proton. Each frequency provides unique characteristics to electron, positron, nucleus, gravity and energy transport in space. Energy transport in space is achieved by energy quanta $\delta \boldsymbol{S}_{v}$ or by superpositioned energy quanta $h v$ (photons) on energy quanta $\delta \boldsymbol{S}_{v_{g}}$.

According to this study electromagnetic theory, quantum physics, elementary particle physics and astrophysics can be described by the same set of rules and laws. The revised version of electromagnetic theory constitutes the fundament for unification of these scientific disciplines into one comprehensive Theory of Everything (TOE).

The novel Theory of Everything will dramatically simplify the description of many natural sciences and ease the understanding of these sciences. Today there are often locked doors between electromagnetic theory, quantum physics, elementary particle physics, astrophysics, bioelectromagnetics, chemistry, biochemistry and medicine. The reason is, among others, that these discipline use different models and different terminology. The Theory of Everything will unify models and terminology. It will result in increased multidisciplinary cooperation and faster progress in R\&D.

The electronics industry rapidly approaches processes and products which operate on only one or few electrons. Understanding the real nature of the electron and the photon can result in a paradigm shift within microelectronics.

Bioelectromagnetics is an important discipline, concerned with the nature of organisms and which has large 
impact on medicine. The Theory of Everything will dramatically change the definition of bioelectromagnetics processes from today's static processes to dynamic electromagnetic processes. It will improve the understanding of the human organism and infectious diseases. It may result in a paradigm shift in medicine.

Many environmental problems are caused by chemicals used in e.g. industrial processes, products and in farming, and where the chemicals eventually are found in the environment, in food and in the human organism. They consist of molecules called PFOS, PFOA, PCB, PBDE, glyphosate, carbendazim, boscalid etc. Molecules are today described as atoms united by the static Coulomb force. In reality the molecule shall be described by the Theory of Everything; energy quanta and oscillator quanta in the atomic nucleus and the electron result in a number of forces which amalgamate atoms into a molecule. This implies that the molecule comprises many dynamic electromagnetic processes. These dynamic electromagnetic processes interact with and disturb dynamic electromagnetic processes in organisms including humans, i.e. the processes described in bioelectromagnetics. Today's static definition of the molecule provides meager understanding of the environmental effects caused by chemicals. In the future it may be possible to disturb the molecule's dynamic processes; it results in that the molecule is destroyed. It may enable remediation of the environment from toxic chemicals. The Theory of Everything may cause a paradigm shift in chemistry and in the understanding of environmental problems.

The earth is exposed to an enormous amount of energy, i.e. energy quanta $\delta \boldsymbol{S}_{v}$. Only a fraction of these energy quanta are absorbed by atoms. The absorbed energy results in nuclear fission and fusion energy used in e.g. nuclear power plants. It may be possible to control this vast amount of energy in the future. There may be no energy problem in the future; there will be endless amount of energy and the energy will be safe with no waste and without environmental impact.

It is proposed that the present study will have major impact on the understanding of basic physics, chemistry and medicine. Hopefully, this will result in multidisciplinary cooperation, faster R\&D, improved products, improved medicine, improved understanding of environmental problems, improved methods to remediate the environment and a solution to the energy and climate problem.

\section{References}

[1] Giertz, H.W. (2013) Open Journal of Microphysics, 3, 115-120.

[2] Giertz, H.W. (2013) International Journal of Astronomy and Astrophysics, 3, 39-50. http://dx.doi.org/10.4236/ijaa.2013.32A007

[3] Giertz, H.W. (2010) Journal of Atmospheric and Solar-Terrestrial Physics, 72, 767-773. http://dx.doi.org/10.1016/j.jastp.2010.03.022

[4] Giertz, H.W. (2013) Open Journal of Microphysics, 3, 71-80. http://dx.doi.org/10.4236/ojm.2013.33013

[5] Giertz, H.W. (2014) Open Journal of Microphysics, 4, 7-14. http://dx.doi.org/10.4236/ojm.2014.42002

[6] Giertz, H.W. (2014) International Journal of Astronomy and Astrophysics, 4, 353-358. http://dx.doi.org/10.4236/ijaa.2014.42029

[7] Giertz, H.W. (2014) International Journal of Astronomy and Astrophysics, 4, 294-300. http://dx.doi.org/10.4236/ijaa.2014.41024

[8] Giertz, H.W. (2014) International Journal of Astronomy and Astrophysics, 4, 384-390. http://dx.doi.org/10.4236/ijaa.2014.42033

[9] Higgs, P.W. (1964) Physical Review Letters, 11, 508-509. http://dx.doi.org/10.1103/PhysRevLett.13.508

[10] Higgs, P.W. (1964) Physical Letters, 12, 132-133. http://dx.doi.org/10.1016/0031-9163(64)91136-9

[11] Englert, F. and Brout, R. (1964) Physical Review Letters, 13, 321-323. http://dx.doi.org/10.1103/PhysRevLett.13.321

[12] Bleaney, B.I. and Bleaney, B. (1965) Electricity and Magnetism. Oxford University Press, Amen House, London.

[13] Weinberg, S. (1972) Gravitation and Cosmology. John Wiley \& Sons, Hoboken.

[14] Okun, L.B. (1989) Physics Today, 42, 31-36. http://dx.doi.org/10.1063/1.881171

[15] Kneubühl, F.K. (1997) Oscillations and Waves. Springer, Berlin. http://dx.doi.org/10.1007/978-3-662-03468-2

[16] Sipe, J.E. (1995) Physical Review A, 52, 1875-1883. http://dx.doi.org/10.1103/PhysRevA.52.1875

[17] Oda, I. (2010) Physics Letters B, 690, 322-327. http://dx.doi.org/10.1016/j.physletb.2010.05.048

[18] Bezrukov, F. and Shaposhnikov, M. (2008) Physics Letters B, 659, 703-706. http://dx.doi.org/10.1016/j.physletb.2007.11.072 
[19] Einstein, A. (1916) Annalen der Physik, 49, 285-339.

[20] Eisberg, R. and Resnick, R. (1985) Quantum Physics of Atoms, Molecules, Solids, Nuclei, and Particles. 2nd Edition, Wiley, Hoboken.

[21] Harris, R. (2008) Modern Physics. 2nd Edition, Pearson International Edition, Addison-Wesley, Boston.

[22] Kurokawa, K. (1973) Proceedings of the IEEE, 61, 1386-1410. http://dx.doi.org/10.1109/PROC.1973.9293

[23] Tiebout, M. (2004) IEEE Journal of Solid-State Circuits, 39, 1170-1174. http://dx.doi.org/10.1109/JSSC.2004.829937

[24] Burgess, C. and Moore, G. (2007) The Standard Model: A Primer. Cambridge University Press, Cambridge.

[25] Polchinski, J. (1998) String Theory. Cambridge University Press, Cambridge. 
Scientific Research Publishing (SCIRP) is one of the largest Open Access journal publishers. It is currently publishing more than 200 open access, online, peer-reviewed journals covering a wide range of academic disciplines. SCIRP serves the worldwide academic communities and contributes to the progress and application of science with its publication.

Other selected journals from SCIRP are listed as below. Submit your manuscript to us via either submit@scirp.org or Online Submission Portal.
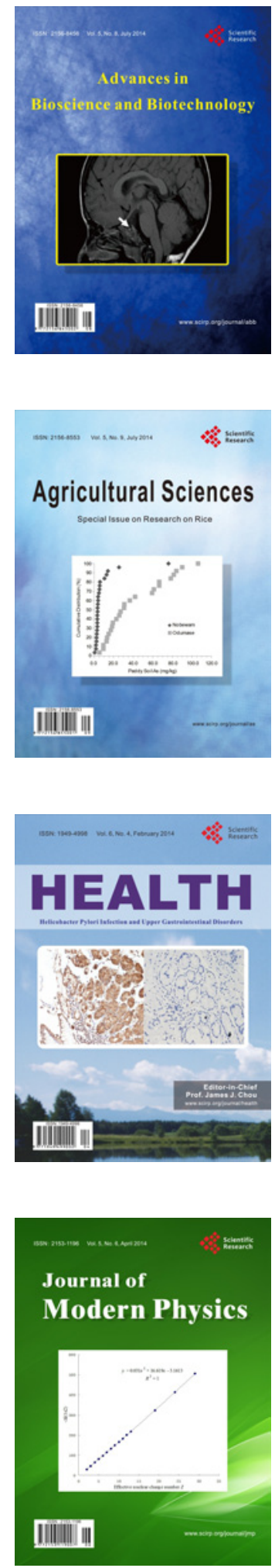
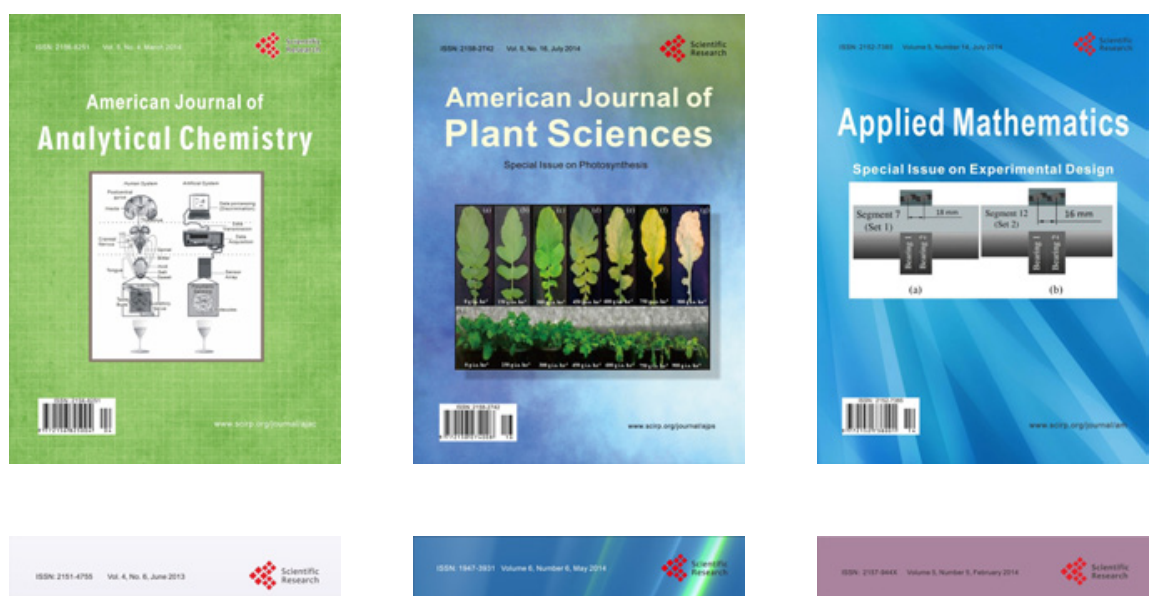

Creative Education
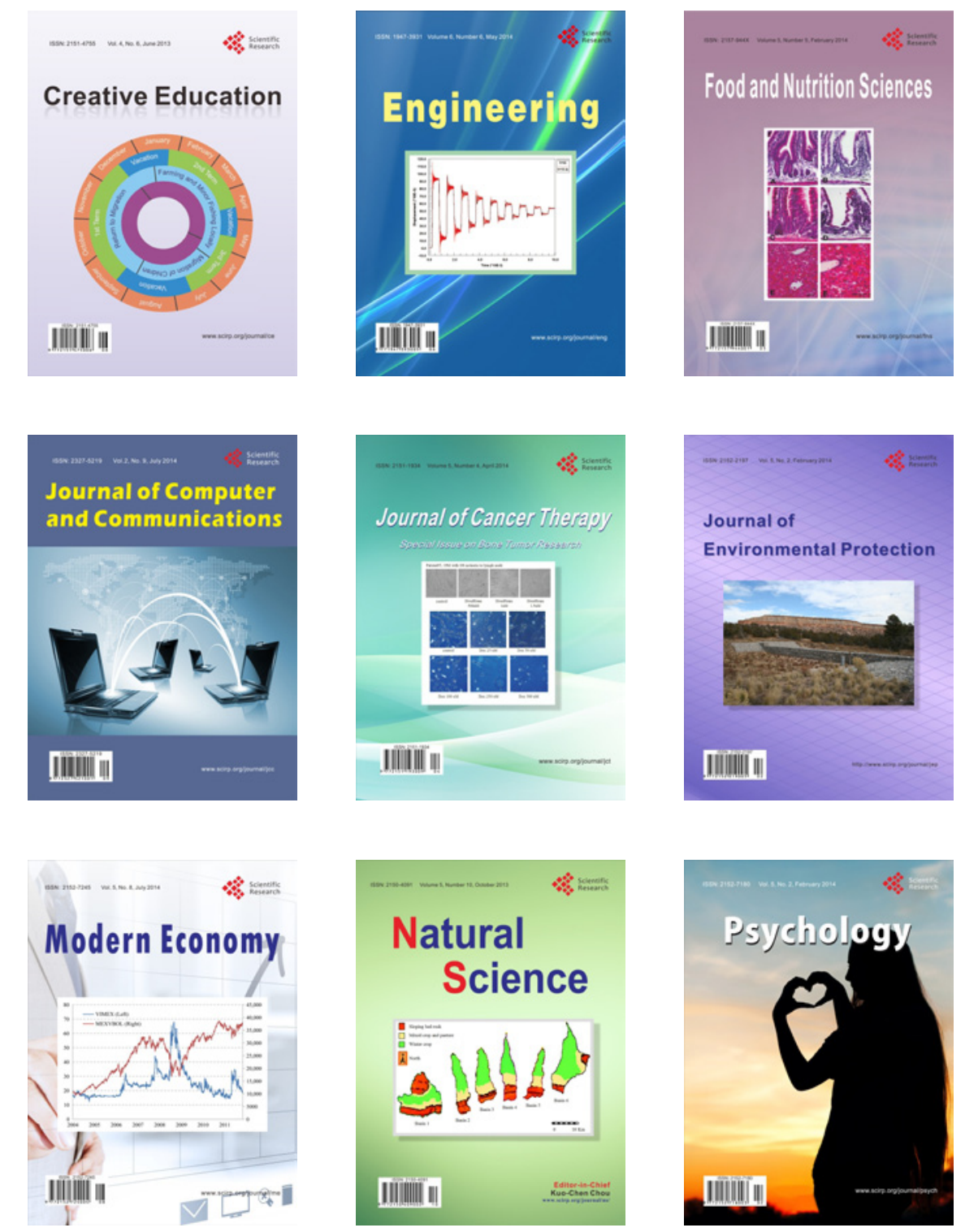